\title{
Environment Modelling for the Robust Motion Planning and Control of Planar Rigid Robot Manipulators
}

\author{
Luca Massimiliano Capisani, Member IEEE Tullio Facchinetti \\ Antonella Ferrara, Senior Member IEEE Alessandro Martinelli \\ Department of Computer Engineering and Systems Science, University of Pavia, ITALY \\ e-mail: \{luca.capisani, tullio.facchinetti, antonella.ferrara, alessandro.martinelli\}@unipv.it
}

\begin{abstract}
Trajectory planning and tracking are crucial tasks in any application using robot manipulators. These tasks become particularly difficult when obstacles are present in the manipulator's workspace. In this paper it is assumed that the obstacles can be approximated in a conservative way with discs. The goal is to represent the obstacles in the robot configuration space, in order to allow an efficient and accurate trajectory planning and tracking. Moreover, the paper provides the methods for checking the collision between the $n$-joint manipulator and the obstacles. Trajectory planning depends on tracking accuracy. In this paper an adequate tracking accuracy is guaranteed assuming the use of a suitably designed robust controller.
\end{abstract}

\section{Introduction}

It is common for robot manipulators to operate in workspaces where the manipulator mobility is limited due to the presence of obstacles. In general, an obstacle can be any object which is present in the robot workspace and must be properly avoided for achieving the correct behaviour of the robot. Other objects may be present in the robot workspace but, depending on the application, they may not be considered as obstacles (e.g., the target object of a grasping device). An application example where obstacles play an important role is the autonomous motion of a robot in a space shuttle, where obstacles are represented by people, tools, instruments and other manipulators [16].

A possible approach to keep in account the presence of obstacles is to analytically describe the obstacles geometry in the manipulator configuration space, and use it for trajectory planning and control. In [5, 13, 14], this approach has been considered together with the problem of planning a suitable trajectory, giving many possible solutions. To plan the trajectory, the geometric description of workspace and the configuration space must be available, even in a partial fashion. However, the description of the configuration space may be particularly complex when the system has more than three degrees of freedom (see $[1,16])$. Some approaches try to avoid the description of the configuration space, by analyzing the shape of the links and the shape of the obstacles directly in the workspace (see, for instance, [11]).

To describe the geometry of obstacles in the configuration space, two fundamental approaches have been followed. The first assumes a model for the whole configuration space before starting the trajectory planning (see $[1,8,9,12,17]$ ) while the second limits the modelling of the configuration space to a region close to the actual robot configuration, by making numerical approximations (see $[16,20]$ ). Since the analytical modelling of the configuration space is, in many cases, complex, numerical approximations are introduced also by relying on fluid dynamics (see $[10,12]$ ).

This paper presents an analytic approach to the description of geometries of planar obstacles, with the aim of developing a dedicated path planning strategy for a robot manipulator. In general, the path planning problem based on a description of the configuration space is solved relying on potential functions or on particular strategies based on the optimization of some cost functions [14]. However, in order to decouple the path planning problem from the problem of trajectory tracking control, robust control methods must be implemented [7]. By adopting a particular Second Order Sliding Mode Control (SOSMC) algorithm [2], it is possible to assume an upper bound for the tracking error. Such upper bound relies on the maximum input disturbance that can occur in the system and depends on the constants used to describe the model.

Despite the complexity of the mapping in the general case, the mapping of circular planar obstacles can be done in an analytical way for a general $n$-link planar manipulator, obtaining interesting and useful geometric properties for the shape of the obstacles in the configuration space. This analysis can be very useful when a 6 d.o.f anthropomorphic manipulator is considered. In fact, in this case, the problem of planning the trajectories for the first three links can be decoupled from the problem of planning the trajectories for the last three links with good approximation, if the lengths of the last 3 links are short enough [15]. Since in an anthropomorphic manipulator the second and the third link make motions on a vertical plane possible, and the first link can be devoted to the selection of such a plane, the geometric analysis of the shape for a planar manipulator becomes relevant. On the other hand, circular obstacles can be thought as a good approximation of various obstacles types, and more 
than one circular obstacle can be defined in order to represent real obstacles having complex generic shapes.

\section{System model and notation}

Throughout the paper, we denote the robot manipulator by $\mathcal{R}$ and its workspace by $\mathcal{W}$. No constraints are supposed on the angular displacements of joints, i.e. it is assumed that each link is able to perform a full revolution around the corresponding joint. All the possible configurations assumed by the robot lie in a space $\mathcal{C}$ called configuration space. A configuration vector is denoted with $Q=\left(Q_{1}, \ldots, Q_{n}\right) \in \mathcal{C}$, where $Q_{i}, 1 \leq i \leq n$, represents the angular displacement of the $i$-th link with respect to the $x$ axis. The $i$-th link has length $l_{i}$ (see Figure 1). Taking into account the manipulator link geometry, $Q$ specifies the position of all points of $\mathcal{R}$ in the space $\mathcal{W}$. The vector $Q(i)=\left(Q_{1}, \ldots, Q_{i}\right) \in \mathcal{C}_{i}, i \leq n$ denotes the $i$-th sub-configuration of $\mathcal{R}$ and the space $\mathcal{C}_{i}$ as a $i$-th sub-configuration space restricted to the first $i$ links.

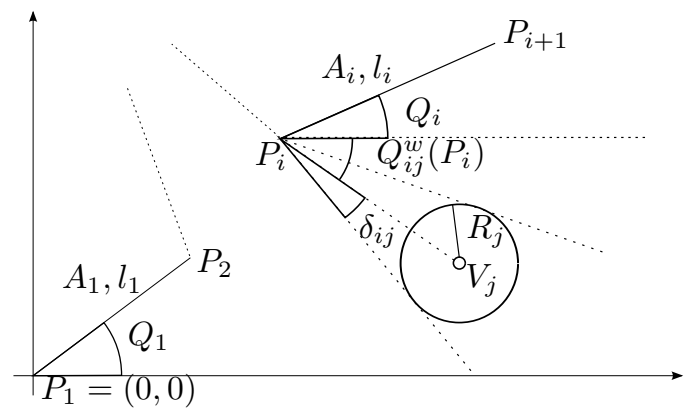

\section{Figure 1. Description of a generic circular ob- stacle and a generic manipulator link in the workspace $\mathcal{W}$.}

The first rotational joint of the manipulator is located at the origin $\mathcal{O}$ of a $\{x, y\}$ plane which represents the workspace $\mathcal{W}$. Given the vector of relative rotations between two consecutive links in the Denavit-Hartenberg representation $q=\left(q_{1}, \ldots, q_{n}\right), Q_{i}$ is

$$
Q_{i}=\sum_{j=1}^{i} q_{j}, \quad i=1, \ldots, n
$$

while the position of the extreme point of each joint $k$ is given by

$$
P_{k}=\left[\begin{array}{l}
x_{k} \\
y_{k}
\end{array}\right]=\sum_{i=1}^{k-1}\left[\begin{array}{c}
l_{i} \cos Q_{i} \\
l_{i} \sin Q_{i}
\end{array}\right]
$$

with $k=2, \ldots, n+1$ and $P_{1} \equiv \mathcal{O}$. The $P_{n+1}$ point represents the position of the end-effector. Moreover it is manifest that the subspaces $\mathcal{C}_{i}$ are $2 \pi$ periodic.

The subset of the points of $\mathcal{W}$ belonging to the robot manipulator when a particular configuration $Q$ is assumed, is denoted by $A(Q) \subseteq \mathcal{W}$. The subset of the points of $\mathcal{W}$ belonging to the $i$-th link of the robot manipulator when a particular configuration $Q$ is assumed, is denoted by $A_{i}(Q) \subseteq$ $\mathcal{W}$. Therefore, $A(Q)=\bigcup_{\forall i} A_{i}(Q)$.
The workspace contains $m$ obstacles denoted by $\mathcal{B}_{j}, 1 \leq$ $j \leq m$, modelled as discs having center $V_{j}$ and radius $R_{j}$; the $j$ term represents the index of the obstacle. The region $\mathcal{W}^{\text {free }}=\mathcal{W}-\cup_{\forall j} \mathcal{B}_{j}$ denotes the subset of workspace points where the robot can be placed without colliding with any obstacle. Each obstacle $\mathcal{B}_{j}$ can be mapped in the subconfiguration space $\mathcal{C}_{i}$ as a subset of points $\mathcal{E B}_{i j}$, i.e.

$$
\mathcal{C B}_{i j}=\left\{Q(i) \in \mathcal{C}_{i}: A_{i}(Q(i)) \cap \mathcal{B}_{j} \neq \emptyset\right\}
$$

indeed, the set $\mathcal{C B}_{i j}$ contains the points representing all the possible configurations in which the $A_{i}$ link collides with the $j$-th obstacle. The subset of $\mathcal{C}_{i}$ containing all the possible configurations in which the $A_{i}$ link can be placed without colliding the obstacles can then be defined as

$$
\mathcal{C}_{i}^{\text {free }}=\mathcal{C}_{i}-\cup_{\forall j} \mathcal{C B}_{i j}
$$

Moreover the free configuration space is denoted by $\mathrm{e}^{\text {free }}=\left\{Q \in \mathcal{C}: \forall i, Q(i) \in \mathcal{C}_{i}^{\text {free }}\right\}$.

The objective of above definitions is to provide the formalism to discuss the approach described in this paper, aiming to find a collision-free path between two configurations denoted by $Q_{\text {init }} \in \mathrm{e}^{\text {free }}$ and a suitable $Q_{\text {goal }} \in \mathrm{e}^{\text {free }}$ representing the desired target to be reached. This path can be formally defined as the problem of finding a continuous map $t: s \rightarrow \mathcal{C}, s \in[0,1], t(s)=\left(t_{1}(s), \ldots, t_{n}(s)\right)$ such that $t(0)=Q_{\text {init }}, t(1)=Q_{\text {goal }}$ and $\forall i, s:\left(t_{1}(s), \ldots, t_{i}(s)\right) \in$ $\mathrm{e}_{i}^{f r e e}$.

\section{Planar $n$-link obstacle mapping}

This section discuss the case of a generic $n$-link planar manipulator with $n$ rotational joints. The purpose is to give an analytical formulation of the shape of obstacles in the configuration space of the manipulator.

\subsection{Collision detection and geometric description of a single obstacle for a single joint}

A single planar circular obstacle $\mathcal{B}$ centered in $V=$ $\left(V_{x}, V_{y}\right)$ with radius $R$ is considered. Since the case of a single obstacle is considered in this section, the $j$ index is left out for simplifying the notation. It is faced the problem of representing the obstacle in a particular sub-configuration space $\mathcal{C}_{i} \in \mathbb{R}^{i}$ and therefore of giving formulas for the geometries of the $\mathcal{C B}_{i}$ subsets.

The two key concepts of critical and worst configuration are now introduced to support the development of the proposed approach.

Definition 1. The critical configuration of a given link $A_{i}$, having the corresponding joint located at $\left[x_{i} ; y_{i}\right]^{T}$, with respect to the obstacle $\mathcal{B}$ centered in $V=\left(V_{x}, V_{y}\right)$ is defined as

$$
Q_{i}^{w} \stackrel{\text { def }}{=} \operatorname{atan} 2\left(V_{x}-x_{i}, V_{y}-y_{i}\right)
$$

The critical configuration, also referred as critical case, for the $A_{i}$ link is defined as the angle for which the link lays on the straight line defined by the obstacle's center and the 
position $P_{i}$ of the $i$-th joint. From Definition 1, it is clear that $Q_{i}^{w}$ is function of $\left(Q_{1}, \ldots, Q_{i-1}\right)$, however such dependency is not reported in the rest of the paper to simplify the notation. Figure 1 shows an example of critical configuration.

Definition 2. The worst configuration $Q^{W}=$ $\left(Q_{1}^{W}, \ldots, Q_{n}^{W}\right)$ for the entire manipulator is when every joint is in its critical configuration.

The worst configuration corresponds to the case in which the versus of each joint points to the center of the obstacle. It is worth to outline the different orientation of each link depending on the relative position of the obstacle with respect to the corresponding link joint.

Looking at Definition 2, it is straightforward to verify that $Q_{1}^{W}=\operatorname{atan} 2\left(V_{x}, V_{y}\right)=Q_{2}^{W}+k_{2} \pi=Q_{3}^{W}+k_{3} \pi=\ldots=$ $Q_{n}^{W}+k_{n} \pi$ for some $k_{i} \in \mathbb{Z}, i=2, \ldots, n$, i.e., all joints lay on the straight line defined by $\mathcal{O}$ and the center of the considered obstacle, and $k_{i}$ are chosen such that the versus of each joint is toward the center of the obstacle.

On the basis of the above definitions, the following result can be proved once defined $\Delta$ as a vector of angular values.

Theorem 3.1. Being $\Delta=\left(\bar{\delta}_{1}, \ldots, \bar{\delta}_{i}\right)$ and $Q^{W}(i)=$ $\left(Q_{1}^{W}, \ldots, Q_{i}^{W}\right)$, the configuration $Q^{W}(i)+\Delta$ causes the $A_{i}$ link to collide with the obstacle $\mathcal{B}$ if and only if also $Q^{W}(i)-\Delta$ does.

Proof. It is straightforward considering the radial symmetry of a circular obstacle with respect to its center and the definition of $Q_{i}^{W}$.

Theorem 3.1 shows that $Q^{W}(i)$ is a point of central symmetry for $\mathcal{C B}_{i}$. An example is shown in Figure 2 in which the property is depicted for two particular angular vectors, namely $\Delta_{A}$ and $\Delta_{B}$.

Theorem 3.1 considers the worst configuration for the $i$ th link. However, the general case corresponds to the situation where the link $A_{i}$ is its critical configuration, while all the $A_{j}, 1 \leq j<i$, are not in their critical configurations. The symmetry property of this latter situation is captured by Theorem 3.2.

Theorem 3.2. Suppose it is given a value $\delta$ and $Q_{i}^{w}$ to be the critical case of $A_{i}$. Then the angle $Q_{i}^{W}+\delta$ causes $A_{i}$ to collide with the obstacle $\mathcal{B}$ if and only if also $Q_{i}^{W}-\delta$ does.

Proof. Again, it is an obvious result of the radial symmetry of circular obstacles and of the definition of critical configuration.

A symmetry property captured by Theorem 3.2 is depicted in Figure 2 for $\mathcal{C B}_{2}$ in which $\delta$ represents a particular angular value.

Definition 3. The angular function $\delta_{i}\left(Q_{1}, \ldots, Q_{i}\right)$ is defined such that when the angular displacement $Q_{i+1}$ of the $A_{i+1}$ link satisfies

$$
Q_{i+1} \in\left(Q_{i+1}^{w}-\delta_{i}(\cdot), Q_{i+1}^{w}+\delta_{i}(\cdot)\right)
$$

the $A_{i+1}$ link intersects the obstacle, otherwise it does not.

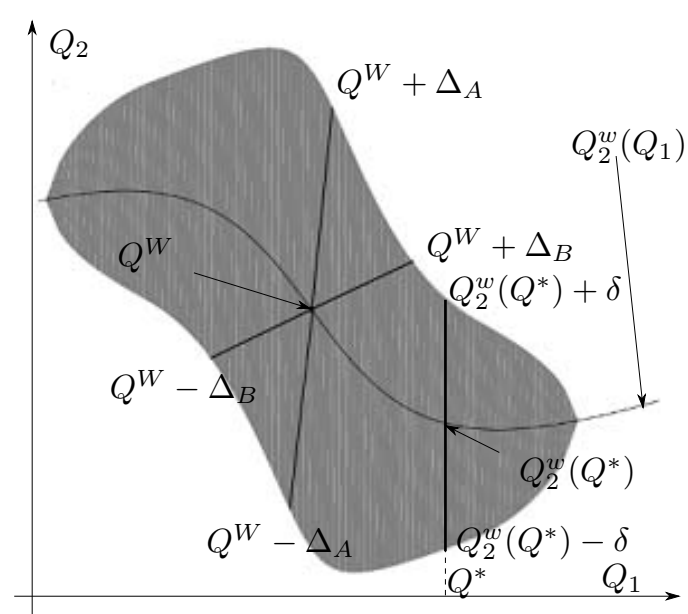

Figure 2. Symmetries of a $\mathrm{CB}_{2}$ geometry. $\Delta_{A}$ and $\Delta_{B}$ are two vectors in $\mathrm{C}_{2}, Q_{1}^{*}$ and $\delta$ are two scalar values.

Theorem 3.3 shows the formulation of the generic $\delta_{i}(\cdot)$ function as a function of the corresponding link, joint and obstacle parameters.

Theorem 3.3. The $\delta_{i}\left(Q_{1}, \ldots, Q_{i}\right)$ function is given by

$$
\delta_{i}(\cdot)=\left\{\begin{array}{c}
\arcsin \frac{R}{d_{i+1}} \quad \text { if } l_{i+1}^{2} \geq d_{i+1}^{2}-R^{2} \\
\arccos \frac{l_{i+1}^{2}+d_{i+1}^{2}-R^{2}}{2 d_{i+1} l_{i+1}} \\
\text { if }\left(d_{i+1}-R\right)^{2}<l_{i+1}^{2} \leq d_{i+1}^{2}-R^{2} \\
0 \quad \text { otherwise }
\end{array}\right.
$$

where the function $d_{i+1}$ is defined as

$$
d_{i+1}\left(Q_{1}, \ldots, Q_{i}\right)=\sqrt{\left(V_{x}-x_{i+1}\right)^{2}+\left(V_{y}-y_{i+1}\right)^{2}}
$$

and $\left[x_{i+1}, y_{i+1}\right]^{T}$ is defined in (2).

Proof. Once $d_{i}$ is defined as the distance from the $i$-th joint to the center of the obstacle, when $l_{i}^{2} \geq d_{i}^{2}-R^{2}$, the link $A_{i}$ can intersect in a tangential way the boundary of the obstacle. In this case, the angular displacement of such tangential intersection is given by $Q_{i}=Q_{i}^{w} \pm \arcsin \frac{R}{d_{i}}$, thus it is independent from $l_{i}$.

When $\left(d_{i}-R\right)^{2}<l_{i}^{2}<d_{i}^{2}-R^{2}, A_{i}$ cannot intersect the boundary of the obstacle in a tangential way. In this case, straightforward trigonometry gives the two angular displacements such that there is only one point of intersection respectively

$$
Q_{i}=Q_{i}^{w} \pm \arccos \frac{l_{i}^{2}+d_{i}^{2}-R^{2}}{2 d_{i} l_{i}}
$$

The third case for the equation (7) is to be considered when the $A_{i+1}$ link cannot reach the obstacle, given the angular displacement $Q_{i}$.

The $\delta_{i}(\cdot)$ function can be used to check a possible collision between the $(i+1)$-th joint and the obstacle, with $i \geq 1$. 
Consider the $\mathcal{C}_{i}^{\text {free }}$ subspace. In case of multiple obstacles, $\mathcal{R}$ does not collide with any $\mathcal{B}_{j}$ if and only if $\left(Q_{1}, \ldots, Q_{i}\right) \in \mathcal{C}_{i}^{\text {free }} \forall i$, i.e.

$$
\forall i, j: Q(i)=\left(Q_{1}, \ldots, Q_{i}\right) \notin \mathrm{eB}_{i, j}
$$

To verify condition (10), each $A_{i+1}$ link of the manipulator must be outside from the obstacle. The possible collision with the link depends, in general, on the configuration of the previous $i$ links. This condition can be written as

$$
\forall i: Q_{i+1} \notin\left[Q_{i+1, j}^{w}-\delta_{i, j}(\cdot), Q_{i+1, j}^{w}+\delta_{i, j}(\cdot)\right]
$$

Note that the complexity required to verify possible collisions is $\mathcal{O}(\mathrm{nm})$, where $n$ is the number of manipulator's links, and $m$ is the number of obstacles in the workspace.

\subsection{Example on a planar 2-link manipulator configura- tion space}

As an example, this section considers the problem of finding the analytical description for the $\mathcal{C B}_{2, j}$ surface, that is the mapping of a planar circular obstacle $\mathcal{B}_{j}$ in the subconfiguration space $\mathcal{C}_{2}$ associated to the $A_{2}$ link of the manipulator.

The planar circular obstacle $\mathcal{B}_{j}$ is centered in $V_{j}=$ $\left(V_{j, x}, V_{j, y}\right)$ with radius $R_{j}$. It is represented by

$$
\left(x-V_{j, x}\right)^{2}+\left(y-V_{j, y}\right)^{2} \leq R_{j}^{2}
$$

The worst configuration is given by

$$
Q_{1, j}^{W}=\operatorname{atan} 2\left(V_{j, x}, V_{j, y}\right)
$$

and $Q_{2, j}^{W}=Q_{1, j}^{W}$ if the center of the obstacle is located outside the reachable area of the first joint, otherwise $Q_{2, j}^{W}=$ $Q_{1, j}^{W}+\pi$.

The generic critical case for the $A_{2}$ link is given by

$$
\begin{aligned}
Q_{2, j}^{w}\left(Q_{1}\right)=\operatorname{atan} 2\left(V_{j, x}-l_{1} \cos \left(Q_{1}\right),\right. & \\
& \left.V_{j, y}-l_{1} \sin \left(Q_{1}\right)\right)
\end{aligned}
$$

The angular function $\delta_{1, j}\left(Q_{1}\right)$ is defined such that when the angular displacement $Q_{2}$ of the $A_{2}$ link satisfies

$$
Q_{2} \in\left[Q_{2, j}^{w}-\delta_{1, j}\left(Q_{1}\right), Q_{i+1}^{w}+\delta_{1, j}\left(Q_{1}\right)\right]
$$

the $A_{2}$ link intersects the obstacle, otherwise it does not. Corollary 3.4 follows from Theorem 7 .

Corollary 3.4. The $\delta_{1, j}\left(Q_{1}\right)$ function is given by

$$
\delta_{1, j}\left(Q_{1}\right)=\left\{\begin{array}{l}
\arcsin \frac{R_{j}}{d_{2, j}} \quad \text { if } l_{2}^{2} \geq d_{2, j}^{2}-R_{j}^{2} \\
\arccos \frac{l_{2}^{2}+d_{2, j}^{2}-R_{j}^{2}}{2 d_{2, j} l_{2}} \\
\text { if }\left(d_{2, j}-R_{j}\right)^{2}<l_{2}^{2} \leq d_{2, j}^{2}-R^{2} \\
0 \quad \text { otherwise }
\end{array}\right.
$$

where

$$
d_{2, j}=\sqrt{\left(V_{j, x}-l_{1} \cos \left(Q_{1}\right)\right)^{2}+\left(V_{j, y}-l_{1} \sin \left(Q_{1}\right)\right)^{2}}
$$

Once the $\delta_{1, j}\left(Q_{1}\right)$ and the $Q_{2, j}^{w}$ functions are defined, the analytical representation of $\mathcal{C B}_{2, j}$ can be written as

$$
\begin{aligned}
& \mathrm{eB}_{2, j}=\left\{\left(Q_{1}, Q_{2}\right) \in \mathcal{C}_{2}:\right. \\
& \left.\quad Q_{2} \in\left(Q_{2, j}^{w}\left(Q_{1}\right)-\delta_{1, j}\left(Q_{1}\right), Q_{2, j}^{w}\left(Q_{1}\right)+\delta_{1, j}\left(Q_{1}\right)\right)\right\}
\end{aligned}
$$

\subsection{Some considerations on the $\mathcal{C B}_{i j}$ geometries}

In this subsection, some characteristics of the $\mathcal{C B}_{i j}$ geometries, especially in the case of multiple obstacles, are described. The following corollaries can be proved considering Equation (7); it is supposed that there are $m$ circular obstacles $\mathcal{B}_{j}$ lying in the $\mathcal{W}$ space, each one related to $n$ geometries $\mathrm{CB}_{i j}$ which are characterized by the functions $Q_{i, j}^{w}\left(Q_{1}, \ldots, Q_{i-1}\right)$ and $\delta_{i-1, j}(\cdot)$ for every $A_{i}$ link.

\subsubsection{Reliance on the obstacle radius}

Suppose that an obstacle $\mathcal{B}_{j}$ is replaced by another obstacle $\mathcal{B}_{j}^{*}$ having radius $R_{j}^{*}$, the same center $\left(V_{j}^{*}=V_{j}\right)$ and $n \mathcal{e} \mathcal{B}_{i j}^{*}$ geometries characterized by a $Q_{i+1, j}^{w, *}$ and a $\delta_{i-1, j}^{*}(\cdot)$ for any $A_{i}$ link.

\section{Corollary 3.5. The following results hold:}

1. if $R_{j}^{*}>R_{j}$, then $Q_{i, j}^{w, *}=Q_{i, j}^{w}$;

2. if $\exists\left(Q_{1}, \ldots, Q_{i-1}\right)$ such that $\delta_{i-1, j}(\cdot)>0$ then $\delta_{i-1, j}^{*}(\cdot)>\delta_{i-1, j}(\cdot)$ and $\delta_{i-1, j}^{*}(\cdot)>0$ for some of values $\left(Q_{1}, \ldots, Q_{i-1}\right)$ in which $\delta_{i-1, j}(\cdot)=0$;

3. $\mathrm{eB}_{i j} \subseteq \mathrm{eB}_{i j}^{*}$.

Corollary 3.5 shows that, when the radius $R^{*}$ is higher than $R$, the curve $Q_{i, j}^{w}$, i.e. the locus of the critical configurations, does not change (item (1)), while the old geometry is a subset of the new one (item (3), which is consequence of item (2)).

Depending on the obstacle radiuses, two interesting situations can be verified, that are illustrated by corollaries 3.6 and 3.7 .

Corollary 3.6. Given the $A_{i}$ link, if $\left\|V_{j}\right\|>R_{j}^{*}+\sum_{k=1}^{i} l_{k}$, then $\mathrm{CB}_{i j} \equiv \emptyset$

Corollary 3.7. Given the $A_{i}$ link, if $\left\|V_{j}\right\|<R_{j}^{*}+\sum_{k=1}^{i-1} l_{k}$, then $\exists \Theta: \Theta \subseteq \mathcal{C}_{i-1}, \quad \delta_{i-1, j}^{*}(\Theta) \geq \pi$

Corollary 3.6 illustrates the situation in which an obstacle is too far and is not reachable by the $A_{i}$ link even in the worst case (see Figure $3 \mathrm{~A}$ ). The second situation represents the cases in which, for some configurations of $Q_{1}, . ., Q_{i-1}$, the $A_{i-1}$ link virtually enters the obstacle and consequently the $A_{i}$ link is inside the obstacle whatever $Q_{i}$ is (see Figure 3 D).

\subsubsection{Reliance on the obstacle position}

Suppose that an obstacle $\mathcal{B}_{j}$ is replaced by another obstacle $\mathcal{B}_{j}^{*}$ having the same radius, but different center, and a $\mathrm{eB}_{i j}^{*}$ characterized by a $Q_{i, j}^{w, *}$ and a $\delta_{i-1, j}^{*}(\cdot)$ for any $A_{i}$ link. Cylindrical coordinates are going to be used to indicate the $\mathcal{B}_{j}$ center $V_{j}=\left(\rho_{j} \cos \left(\theta_{j}\right), \rho_{j} \sin \left(\theta_{j}\right)\right)$ and the $\mathcal{B}_{j}^{*}$ center $V_{j}^{*}=\left(\rho_{j}^{*} \cos \left(\theta_{j}^{*}\right), \rho_{j}^{*} \sin \left(\theta_{j}^{*}\right)\right)$.

Corollary 3.8. The critical case functions for the obstacle $\mathcal{B}_{j}^{*}$ are $Q_{i, j}^{*, w}=Q_{i, j}^{w}+\left(\theta_{j}^{*}-\theta_{j}\right), \forall i$. If $\rho_{j}=\rho_{j}^{*}$, then, the $\mathrm{CB}_{i j}^{*}$ geometries are obtained from the $\mathrm{CB}_{i j}$ geometries by means of a rigid translation in $\mathrm{C}_{i}$ of a vector $\left(\theta_{j}^{*}-\theta_{j}, \theta_{j}^{*}-\right.$ $\left.\theta_{j}, \ldots, \theta_{j}^{*}-\theta_{j}\right)$. 


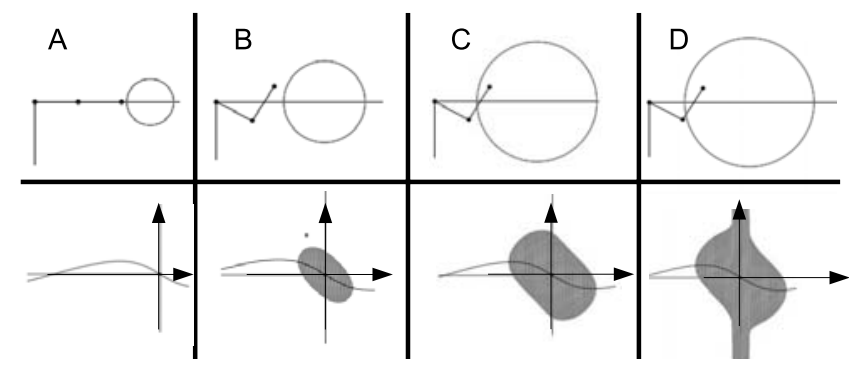

Figure 3. $\mathcal{W}$ and $C_{2}$ spaces when the radius is changed: $(A)$ The radius is small enough, then $\mathrm{CB}_{2 j}=\emptyset$; (B-C) $\mathrm{CB}_{2 j}$ is not a null set; (D) The radius is large and, for some values of $Q_{1}$, any value of $Q_{2}$ causes a collision.

If $\rho_{j}<\rho_{j}^{*}$ then the shapes of geometries $\mathcal{C B}_{i j}^{*}(\forall i)$ change: the new shapes can be evaluated with considerations very closed to the ones seen in the case of changing the radius of an obstacle.

\subsubsection{Reliance on the link length}

Suppose that the $A_{i}$ link is replaced by a different link having length $l_{i}^{*}$. Define $\Psi_{i j}(l): \mathbb{R} \rightarrow \mathcal{C}_{-}$as the set of sub-configurations $\left(Q_{1}, \ldots, Q_{i-1}\right)$ in which $l^{2} \geq$ $\left(d_{i}\left(Q_{1}, \ldots, Q_{i-1}\right)\right)^{2}-R_{j}^{2}$ (see equation (8)).

Corollary 3.9. 1. If $l_{i}^{*}>l_{i}$ then $\Psi_{i j}\left(l_{i}\right) \subseteq \Psi_{i j}^{*}\left(l_{i}^{*}\right)$. For these configurations, the intersections analysis does not depend on the link length.

2. If $\Psi_{i j}^{*}\left(l_{i}^{*}\right) \equiv \mathcal{C}_{i-1}$, then $\delta_{i j}^{*}(\cdot)=\arcsin \frac{R_{j}}{d_{i}\left(x_{i}, y_{i}\right)}>$ $0 \forall\left(Q_{1}, \ldots, Q_{i-1}\right) \subseteq \mathcal{C}_{i-1}$.

Item (2) states that a long link may create a partition of the the $\mathcal{C}_{i}$ space. This partition means that the manipulator cannot step over an obstacle turning completely around it. If the partitioning obstacles are more than one, the manipulator may not be able to reach some feasible configurations (Figure 4).

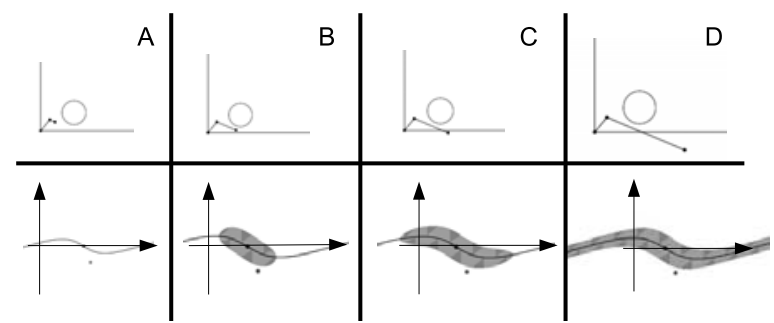

Figure 4. $\mathcal{W}$ and $C_{2}$ spaces when the second link length is changed: (A) The link is short, $\mathrm{CB}_{2 j}=\emptyset$; (B-C) The $\mathrm{eB}_{2 j}$ is not a null set; (D) The link is long and, for any values of $Q_{1}$, there are some values of $Q_{2}$ which causes a collision (a partitioning is present, $\Psi_{2 j} \equiv \mathcal{C}_{1}$ ).

\subsubsection{Multiple Obstacles}

When more than one obstacle are present, some considerations can be done (see Figure 5).

Corollary 3.10. The worst configuration for the $A_{i}$ link is always on one of these special directions: $Q_{i j}^{W}=Q_{1 j}^{W}+$ $k \pi, \forall i, j, k \in \mathbb{Z}$.

Having multiple obstacles, a remarkable situation is when two of the geometries $\mathcal{C B}_{i j}$ intersect each other. This situation is very important because it indicates the case in which it is impossible to turn around an obstacle without collide with the other, and may induce problems during the trajectory planning. For this reason in the next subsection we will discuss the problem of identifying these intersections.

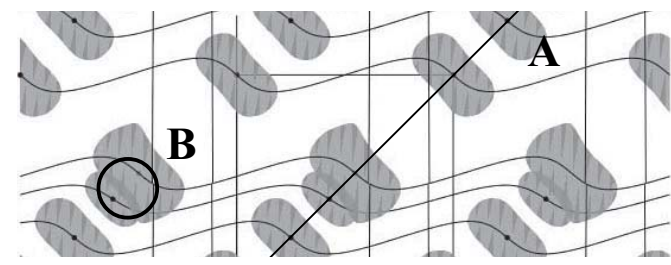

Figure 5. Multiple Obstacles $\left(\mathcal{C B}_{2 j}\right)$ : (A) all the $\mathrm{CB}_{i j}$ are located with the central symmetry center on the same straight line; (B) Two geometries can intersect in some points.

\subsubsection{Intersections of the $\mathcal{C B}_{i j}$ geometries}

The problem of finding the intersections of geometries $\mathrm{CB}_{i j}$ can be solved by evaluating the non-linear system made by the equations which describe the geometries using numerical methods. However, the formulas change from link to link (every aspect of the $A_{i}$ link is solved in $\mathrm{C}_{i}$ ); in this section some considerations are given which can simplify some aspects of the evaluation.

Definition 4. It is given an obstacle $\mathcal{B}_{j}$. Suppose that the generic $A_{i}$ link has its first point in $P_{i}=\left(x_{i}(\cdot), y_{i}(\cdot)\right)$, length $l_{i}$ and direction given by $Q_{i}$. The function $d_{i j}\left(x_{i}(\cdot), y_{i}(\cdot)\right)$ is the distance between $V_{j}$ and $P_{i}$. The $c i r-$ cular equivalent obstacle $\mathcal{B}_{i j}\left(x_{i}(\cdot), y_{i}(\cdot)\right)$ is defined as the obstacle having center $V_{i j}^{\prime}\left(x_{i}, y_{i}\right)$ and radius $R_{i j}^{\prime}\left(x_{i}, y_{i}\right)$ so that $\left(V_{i j}^{\prime}-P_{i}\right) d_{i}=\left(V_{j}-P_{i}\right)$ and the radius is to be determined in one of this two ways: if $l_{i}^{2} \geq d_{i j}^{2}-R_{j}^{2}$ then $R_{j}^{\prime}=\frac{R_{j}}{d_{i j}}$, else $R_{j}^{\prime}=\sqrt{1-\left(\frac{l_{i}^{2}+d_{i j}^{2}-R_{j}^{2}}{2 d_{i j} l_{i}}\right)^{2}}$.

From the definition of $V_{i j}^{\prime}$ it is obvious that $d_{i-1, j}^{\prime}\left(x_{i}, y_{i}\right)=\left\|V_{i j}^{\prime}-P_{i}\right\|=1$. The locally defined circular equivalent obstacle depends on the starting point $P_{i}\left(Q_{1}, \ldots, Q_{i-1}\right)$, which is the reason to say it is locally defined. Theorem 3.11 explains the equivalence.

Theorem 3.11. The $A_{i}$ link starting at point $P_{i}\left(Q_{1}, \ldots, Q_{i-1}\right)$ intersects $\mathcal{B}_{j}$ if and only if it intersects the locally defined circular equivalent obstacle $\mathcal{B}_{i, j}^{\prime}\left(Q_{1}, \ldots, Q_{i-1}\right)$ 
Proof. The proof follows from Theorem 3.3. In fact $R_{j}^{\prime}$ is evaluated so that $\delta_{i j}=\arcsin R_{j}^{\prime}$ in every case. The function $\delta_{i j}^{\prime}$ can be evaluated as $\delta_{i j}^{\prime}=\arcsin \frac{R_{j}^{\prime}}{d_{i}^{\prime} j\left(x_{i}, y_{i}\right)}$, but $d_{i j}^{\prime}\left(x_{i}, y_{i}\right)=1$. Then $\delta_{i j}=\delta_{i j}^{\prime}$

The result of Theorem 3.11 gives the possibility of proposing a simple rule to characterize the intersections of the geometries.

Theorem 3.12. Given the $A_{i}$ link, two different obstacles $\mathcal{B}_{j}$ and $\mathcal{B}_{k}$, and a sub-configuration $\left(Q_{1}, \ldots, Q_{i-1}\right)$, if $R_{j}^{\prime}+R_{k}^{\prime} \geq d_{i(j, k)}^{\prime}$, where $d_{i(j, k)}^{\prime}=\left\|V_{i j}^{\prime}-V_{i k}^{\prime}\right\|$, then the interval of values $Q_{i}$ for which the link intersects both $\mathcal{B}_{j}$ and $\mathcal{B}_{k}$ is non-null.

Proof. The locally defined circular equivalent obstacles $\mathcal{B}_{j}^{\prime}$ and $\mathcal{B}_{k}^{\prime}$ are considered. The center of these discs, i.e. $V_{i j}^{\prime}$ and $V_{i k}^{\prime}$, are at distance 1 from $\left(x_{i}, y_{i}\right)$. The triangle given by $\left(x_{i}, y_{i}\right), V_{i j}^{\prime}$ and $V_{i k}^{\prime}$ is an isosceles triangle. If $R_{j}^{\prime}+R_{k}^{\prime} \geq d_{i(j, k)}^{\prime}$, then it is impossible to trace a ray between $\left(x_{i}, y_{i}\right)$ and any point on the edge given by $V_{i j}^{\prime}$ and $V_{i k}^{\prime}$ without intersecting one of the the two locally defined circular equivalent obstacles, i.e. without intersecting one of the real obstacles.

\section{Planar $n$-link trajectory planning with mul- tiple obstacles}

In this section the problem of the existence of a collision free path between two configurations for a $n$-link robot manipulator is considered. A particular solution is proposed in the 2 -link case. The problem of path planning for a $n$-link manipulator can be solved considering known heuristic algorithms [5]. The geometric description given by previous sections is useful to determine the best solution to the path planning problem.

\subsection{Goal point reachability}

As stated in Section 2, the problem of finding a suitable path between an initial configuration $Q_{\text {init }}=$ $\left(Q_{\text {init } 1}, Q_{\text {init } 2}\right) \in \mathrm{e}^{\text {free }}$ and a final configuration $Q_{\text {goal }}=$ $\left(Q_{\text {goal } 1}, Q_{\text {goal } 2}\right) \in \mathrm{e}^{\text {free }}$ is the problem of finding a continuous function $t(s)$ connecting $Q_{\text {init }}$ to $Q_{\text {goal }}$ which represents a collision free set of configurations. Some considerations are needed to prove the main result regarding the goal point reachability.

Definition 5. The minimal burden of the robot is obtained by optimizing the function

$$
\begin{gathered}
\min _{\left\{a_{i}\right\}}\left\{\left|\inf \mathcal{S}\left(a_{2}, \ldots, a_{n}\right)\right|,\left|\sup \mathcal{S}\left(a_{2}, \ldots, a_{n}\right)\right|\right\} \\
a_{i}= \pm 1, i=2, \ldots, n
\end{gathered}
$$

where

$$
\mathcal{S}(\cdot)=\left\{l_{1}, l_{1}+a_{2} l_{2}, \ldots, l_{1}+\sum_{p=2}^{n} a_{p} l_{p}\right\}
$$

Definition 6. Given an angular displacement for $Q_{1}$, a minimal burden configuration for the robot $\mathcal{R}$ is given by

$$
\left(Q_{1}, a_{2} Q_{1}, a_{3} Q_{1}, \ldots, a_{n} Q_{1}\right)
$$

where $a$ vector minimizes (18).

A fundamental condition to be evaluated in order to guarantee the existence of a path is given by

$$
\bigcup_{\forall j, k}\left(\mathcal{C B}_{i j} \cap \mathcal{C} \mathcal{B}_{i k}\right)=\emptyset
$$

This condition assures that each $A_{i}$ link cannot collide at the same time with two different obstacles.

Finally, the main result reported in this paper regarding the existence of a path is given by the following theorem.

Theorem 4.1. Given $m$ circular obstacles $\mathcal{B}_{j}, 1 \leq j \leq m$, in the workspace $\mathcal{W}$ and a planar n-link robot manipulator $\mathcal{R}$, if

- the robot, at any minimal burden configuration, can move without colliding any obstacle.

- there are no intersections of the $\mathrm{CB}_{i j}$ geometries in the e space, i.e.

$$
\bigcup_{\forall j, k}\left(\mathcal{C B}_{i j} \cap \mathcal{C} \mathcal{B}_{i k}\right)=\emptyset
$$

then a collision-free trajectory between two configurations $Q_{\text {init }} \in \mathrm{e}^{\text {free }}$ and $Q_{\text {goal }} \in \mathrm{e}^{\text {free }}$ is guaranteed to exist.

Proof. A collision free trajectory in the configuration space between two configurations $Q_{\text {init }} \in \mathcal{C}^{\text {free }}$ and $Q_{\text {goal }} \in$ $\mathrm{e}^{\text {free }}$ can always be found if the $\mathcal{C}^{\text {free }}$ space is completely connected. Note also that the $\complement^{\text {free }}$ space is periodic in all the dimensions for a robot manipulator.

In order to exclude the presence of non-connected subsets of the $\mathrm{C}^{\text {free }}$ space, since, by the first condition, the $\mathrm{CB}_{i j}$ geometries are limited and simply connected subsets (see Subsection 3.3.3), it has to be avoided the presence of intersections between two different $\mathrm{CB}_{i}$ geometries which can lead to the presence of partitioning of the space $\mathcal{C}^{\text {free }}$. Since the best work conditions for the robot are given by the minimal burden configuration, then if the robot can move around the origin remaining in the minimal burden configuration, no more partitioning of the space $\mathrm{e}^{\text {free }}$ is present.

\subsection{Two link trajectory planning}

In this section it is considered the problem of planning a trajectory for a 2-link manipulator $\mathcal{R}$ moving in a workspace $\mathcal{W}$ with $m$ obstacles $\mathcal{B}_{j}$. Assume that the conditions of Theorem 4.1 hold, then the two joints of the manipulator cannot reach the obstacles. In this case, the hypothesis of Theorem 3.1 are verified and all the obstacles have theirs center of symmetry lying on the straight line $Q_{1}=Q_{2}$, which is defined in the $\mathcal{C}$ space. 
Two points in the configuration space, given by the following two systems, are useful for defining the initial stage of the path

$$
\begin{aligned}
Q^{\prime} & =\left\{\begin{array}{l}
Q_{2}=Q_{1}+\pi \\
Q_{2}=Q_{i n i t 2}-\left(Q_{1}-Q_{i n i t 1}\right)
\end{array}\right. \\
Q^{\prime \prime} & =\left\{\begin{array}{l}
Q_{2}=Q_{1}-\pi \\
Q_{2}=Q_{i n i t 2}-\left(Q_{1}-Q_{i n i t 1}\right)
\end{array}\right.
\end{aligned}
$$

while the following two points can be useful for the final stage of the path

$$
\begin{aligned}
Q^{*} & =\left\{\begin{array}{l}
Q_{2}=Q_{1}+\pi \\
Q_{2}=Q_{\text {goal } 2}-\left(Q_{1}-Q_{\text {goal } 1}\right)
\end{array}\right. \\
Q^{* *} & =\left\{\begin{array}{l}
Q_{2}=Q_{1}-\pi \\
Q_{2}=Q_{\text {goal } 2}-\left(Q_{1}-Q_{\text {goal } 1}\right)
\end{array}\right.
\end{aligned}
$$

The path to be followed can be obtained in the following way:

- move the robot from the initial configuration $Q_{\text {init }}$ to the closer point between $Q^{\prime}$ and $Q^{\prime \prime}$ along a straight line defined in the $\mathcal{C}$ space.

- move the robot from the current configuration to the closer point between $Q^{*}$ and $Q^{* *}$ along a straight line.

- move the robot from the current configuration to the $Q_{\text {goal }}$ configuration along a straight line.

It is still possible that, applying the foregoing procedure, the robot collides with an obstacle. In this case, the following rule can be introduced.

- Define, for each obstacle $\mathcal{B}_{j}$, a security area (which can be considered as an area obtained by enlarging a particular $\mathcal{C B}_{i j}$ by a particular angular value) such that it is not possible, by considering the performances of the robot controller, to hit the obstacle after the robot has accidentally entered the security area of a particular obstacle.

- Check, for each sampling instant, if any link of the manipulator entered the security area of an obstacle.

- If the robot configuration has entered in any security area $\mathcal{C} \mathcal{B}_{i j}^{*}$, follow the geometry of the security area until the trajectory intersects the straight line that was followed by the trajectory before entering the security area.

Moreover, the check needed to choose the trajectory can be done by considering the $\delta_{i, j}$ functions (see Subsection 3.1) with little computational effort.

\section{Tracking accuracy analysis}

In order to guarantee collision free motions of a real robot manipulator $\mathcal{R}$, a suitable controller must be implemented. This controller has to accurately track the planned trajectory $q_{d}(t) \in \mathbb{R}^{n}$, provided that the tracking represents a feasible goal, i.e. accelerations $\ddot{q}_{d}$ are bounded and positions $q_{d}$ and velocities $\dot{q}_{d}$ do not exceed the limits imposed by the mechanical constraints.

By considering classical manipulator control algorithms, such as PID [18] and adaptive control laws [6], it may be difficult to guarantee an exact tracking of a reference trajectory, since the project of these controllers do not take in account the tracking error as a main objective to be achieved. The main problem is represented by the presence, in the controlled system, of noise, uncertainties, unmodelled effects, and unknown payloads, which can lead to a significant deviation from the expected behaviour of the system dynamics. However in real experiments, due to uncertainties, an exact tracking cannot be guaranteed since common digital controllers have a finite sampling frequency. Finally, note that also bounded small biases on sensor measurements can be interpreted as bounded tracking errors.

A possible solution to this problem, almost in the ideal case (i.e. when infinite sampling frequency of the controller is assumed), is represented by sliding mode control laws [19]. These control laws are robust versus input uncertainties, unmodelled effects, and unknown payloads. In presence of finite sampling time, the discretization chatter effect becomes evident, but the tracking error remains bounded and this bound depends on the maximum uncertainties and on the sampling time [3]. To reduce the discretization chatter effect, higher order sliding mode control algorithms can be adopted, as in [4]. Note that since the bound on the tracking error exists, it can be found projecting the controller or by means of experimental evaluations.

Suppose that a sliding mode controller is adopted to control a $n$-link planar manipulator $\mathcal{R}$ and consequently the maximum tracking error is represented by $\Delta e=$ $\left(\Delta e_{1}, \ldots, \Delta e_{n}\right)$ where $\Delta e_{i}=\sup \left(\left|q_{d i}(t)-q_{i}(t)\right|\right)$. This tracking error leads to an uncertain positioning of the whole manipulator. In this case, given the nominal (i.e. desired) configuration $q_{d}$, the real configuration $\hat{q}_{d}$ lies in the interval

$$
\hat{q}_{d i}=\left[q_{d i}-\Delta e_{i} ; q_{d i}+\Delta e_{i}\right] \forall i
$$

hence, the position $\hat{Q}_{d}$ in the space $\mathcal{C}$ lies in the interval

$$
\hat{Q}_{d i}=\left[Q_{d i}-\sum_{j=1}^{i} \Delta e_{j} ; Q_{d i}+\sum_{j=1}^{i} \Delta e_{j}\right] \forall i
$$

In this case, once defined $\Delta E_{i}=\sum_{j=1}^{i} \Delta e_{j}$, the maximum distance between the expected position $Q$ and the real position $\hat{Q}$ is given by

$$
\sup \sqrt{(Q-\hat{Q})^{T}(Q-\hat{Q})}=\sup \sqrt{\Delta E^{T} \Delta E}
$$

The maximum tracking error expressed by (27) is useful to define a security area around the obstacle to ensure collision-free operations.

\section{A simulation example}

Consider a 2-link manipulator with $l_{1}=0.5 \mathrm{~m}$ and $l_{2}=$ $1 \mathrm{~m}$. Two circular obstacles are present in the workspace, the first with center $V_{1}=(0.62 ; 0.66) \mathrm{m}$ and radius $R_{1}=0.22 \mathrm{~m}$ 
and the second with center $V_{2}=(1.3 ;-0.9) \mathrm{m}$ and radius $R_{2}=0.62 \mathrm{~m}$ (see Figure 6 ). A security area is considered surrounding the $\mathcal{C B}_{i j}$ geometries. The initial configuration is $Q_{\text {init }}=A=(-78 ;-67.6) \mathrm{deg}$ (see Figure $6 \mathrm{~A}$ ) while the final configuration is $Q_{\text {goal }}=D=(8.02 ; 37.82) \mathrm{deg}$ (see Figure $6 \mathrm{D}$ ). The complete path is composed by three stages. First the robot moves to the point B, then the robot moves along the straight line $Q_{2}=Q_{1}+\pi$ reaching the point C. Finally, the robot moves to $\mathrm{D}$ along the straight line $Q_{2}=0.66-\left(Q_{1}-0.14\right)$ until the security area (marked with the light gray color in figure) of the obstacle is reached. The security area of the obstacle is followed and finally the robot moves to the point $\mathrm{D}$. The security area of the obstacle is obtained by assuming a maximum tracking error of $0.1 \mathrm{deg}$ for the two joints.

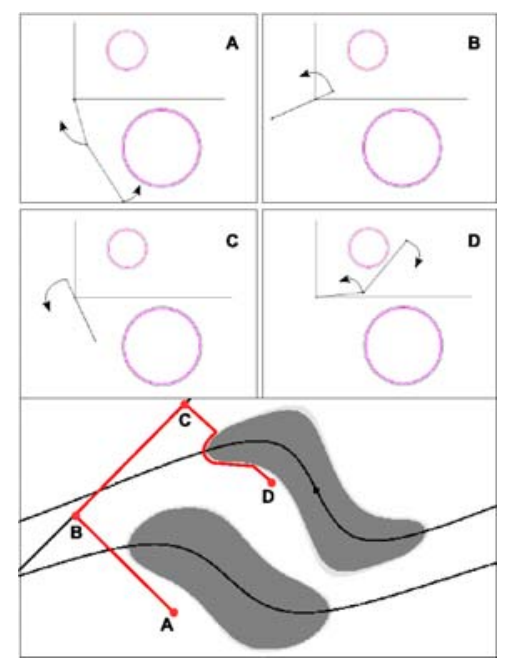

Figure 6. $\mathcal{W}$ and $\mathcal{C}_{2}$ in the simulation example.

\section{Conclusions}

This paper describes the geometric modellization of circular planar obstacles in the configuration space of a $n$-links manipulator. The analytical description of the obstacle geometries is done to allow an accurate trajectory planning and tracking.

The properties of the proposed model are discussed when main parameters, like the length of links and obstacles, are changed. The paper also provides a method to check the collision between the manipulator and obstacles, and to efficiently plan the robot trajectory in presence of multiple obstacles within the workspace. The special case of a 2-link manipulator is described in details and an example of path planning is given for that case.

\section{References}

[1] J. Barraquand, B. Langlois, and J. Latombe. Numerical potential field techniques for robot path planning. IEEE Transactions on Systems, Man, and Cybernetics, 22(2):224-241, Mar./Apr. 1992.
[2] G. Bartolini, A. Ferrara, and E. Usai. Chattering avoidance by second order sliding mode control. IEEE Transactions on Automatic Control, 43(2):241-246, Feb. 1998.

[3] A. Calanca, L. M. Capisani, A. Ferrara, and L. Magnani. An inverse dynamics-based discrete-time sliding mode controller for robot manipulators. In K. Kozlowski, editor, Robot Motion and Control 2007, pages 137-146. Springer-Verlag, London, UK, 2007.

[4] L. M. Capisani, A. Ferrara, and L. Magnani. Design and experimental validation of a second order sliding-mode motion controller for robot manipulators. International Journal of Control, to appear, 2008.

[5] H. Choset, K. M. Lynch, S. Hutchinson, G. Kantor, W. Burgard, L. E. Kavraki, and S. Thrun. Principles of Robot Motion, Theory, Algorithms, and Implementations. MIT Press, Cambridge, Massachusetts, USA, 2005.

[6] R. D. Colbaugh, E. Bassi, F. Benzi, and M. Trabatti. Enhancing the trajectory tracking performance capabilities of position-controlled manipulators. In Proc. IEEE Industry Applications Conference, volume 2, pages 1170-1177, Rome, Italy, Oct. 2000.

[7] A. Ferrara and M. Rubagotti. Gradient tracking based second order sliding mode control of a wheeled vehicle. In Proc. European Control Conference, Kos, Greece, Jul. 2007.

[8] J. Guldner, V. I. Utkin, H. Hashimoto, and F. Harashima. A potential field approach to path planning. In Proc. IEEE Conference on robotics and automation, volume 3, pages 30513056, Nagoya, Aichi, Japan, Feb. 1995.

[9] Y. K. Hwang and N. Ahuja. A potential field approach to path planning. IEEE Transactions on Robotics and Automation, 8(1):23-31, Feb. 1992.

[10] D. Keymeulen and J. Decuyper. The fluid dynamics applied to mobile robot motion: the stream field method. In Proc. IEEE Conference on Robotics and Automation, volume 1, pages 378-385, San Diego, California, USA, May. 1994.

[11] O. Khatib. Real-time obstacle avoidance for manipulators and mobile robots. The International Journal of Robotics Research, 5(1):90-98, Jan. 1986.

[12] J. O. Kim and P. K. Kosla. Real-time obstacle avoidance using harmonic potential functions. IEEE Transactions on Robotics and Automation, 8(3):338-349, Jun. 1992.

[13] J. C. Latombe. Robot Motion Planning. Kluwer Academic Publishers, Dordrecht, The Netherlands, 1991.

[14] S. M. LaValle. Planning algorithms. Cambridge University Press, Cambridge, Massachusetts, USA, 2006.

[15] T. Lozano-Pérez. A simple motion-panning algorithm for general robot manipulators. IEEE Journal of Robotics and Automation, RA-3(3):224-238, Jun. 1987.

[16] E. Ralli and G. Hirzinger. Fast path planning for robot manipulators using numerical potential fields in the configuration space. In Proc. IEEE Intelligent Robots and Systems, pages 1922-1929, Munich, Germany, Sep. 1994.

[17] E. Rimon and D. E. Koditschek. Exact robot navigation using artificial potential functions. IEEE Transactions on Robotics and Automation, 8(5):501-518, Oct. 1992.

[18] L. Sciavicco and B. Siciliano. Modelling and Control of Robot Manipulators. Springer-Verlag, London, UK, second edition, 2000.

[19] V. I. Utkin. Sliding modes in control and optimization. Springer-Verlag, Moscow, Russia, 1992.

[20] J. Zelek. Dynamic path planning. In Proc. IEEE Conference on Systems, Man and Cybernetics, pages 1285-1290, Vancouver, British Columbia, Canada, Oct. 1995. 